R. Hermann · K. Lipponen • M. Kiviniemi • T. Kakko •

R. Veijola $\cdot$ O. Simell $\cdot$ M. Knip $\cdot$ J. Ilonen

\title{
Lymphoid tyrosine phosphatase (LYP/PTPN22) Arg620Trp variant regulates insulin autoimmunity and progression to type 1 diabetes
}

Received: 19 October 2005 / Accepted: 13 February 2006 / Published online: 14 April 2006

C) Springer-Verlag 2006

\begin{abstract}
Aims/hypothesis: We analysed the contribution of the lymphoid protein tyrosine phosphatase (LYP) Arg620Trp variant (which corresponds to the PTPN22 C1858T polymorphism) to the emergence of beta-cellspecific humoral autoimmunity and progression to type 1 diabetes in man. We also explored the heterogeneity in the disease-predisposing effect of this polymorphism in relation to known disease loci, sex and age at disease onset. Subjects and methods: A population-derived Finnish birth cohort with increased disease susceptibility conferred by $H L A-D Q B 1$ was monitored for the appearance of islet cell
\end{abstract}

R. Hermann $\cdot$ K. Lipponen $\cdot$ M. Kiviniemi $\cdot$ T. Kakko $\cdot$ R. Veijola · O. Simell · M. Knip · J. Ilonen JDRF Centre for Prevention of Type 1 Diabetes in Finland, Turku, Finland

R. Hermann $(\bowtie) \cdot$ K. Lipponen $\cdot$ M. Kiviniemi

T. Kakko $\cdot$ J. Ilonen

Department of Virology, University of Turku,

Kiinamyllynkatu 13,

20520 Turku, Finland

e-mail: robert.hermann@utu.fi

Tel.: +358-2-3337023

Fax: $+358-2-3337000$

R. Hermann

Immunogenomics Laboratory,

CellScreen Applied Biomedical Research Center,

Semmelweis University,

Budapest, Hungary

R. Veijola

Department of Paediatrics, University of Oulu,

Oulu, Finland

O. Simell

Department of Paediatrics, University of Turku,

Turku, Finland

M. Knip

Department of Paediatrics, Tampere University Hospital,

Tampere, Finland

M. Knip

Hospital for Children and Adolescents, University of Helsinki,

Helsinki, Finland autoantibodies, and individuals found to be positive were tested for autoantibodies against insulin (IAA), glutamic acid decarboxylase and islet antigen-2 $(n=574$; mean follow-up time 4.9 years). Gene interaction effects on disease susceptibility were analysed in case-control and family series (546 patients, 538 controls, 245 nuclear families). All subjects were typed for HLA $D R-D Q$, insulin gene (INS), CTLA4 and PTPN22 C1858T polymorphisms. Results: The PTPN22 1858TT genotype was associated with the appearance of IAA (adjusted hazard ratio $=4.6,95 \%$ CI $2.4-9.0 ; p=0.000013)$. PTPN22, INS and $H L A-D R B 1$ had an additive effect on the emergence of IAA. The 1858TT and CT genotypes conferred an increased risk of developing additional autoantibodies or clinical disease (hazard ratio $=4.1,95 \%$ CI 1.5-11.6; and $1.6,95 \%$ CI $1.1-2.4$, respectively; $p=0.003$ ). The strong effect of PTPN22 on disease susceptibility $\left(p=2.1 \times 10^{-8}\right)$ was more pronounced in males $(p=0.021)$ and in subjects with non- $D R 4-D Q 8 /$ low-risk HLA genotypes $(p=0.0004)$. Conclusions/interpretation: In the pathogenesis of type 1 diabetes the underlying mechanism of the PTPN22 C1858T polymorphism appears to involve regulation of insulin-specific autoimmunity. Importantly, it strongly affects progression from prediabetes to clinical disease.

Keywords Autoantibodies · Autoimmune disease · Autoimmunity · CTLA4 - DQB1 · DRB1 · HLA · Insulin · Insulin gene $\cdot P T P N 22 \cdot$ Type 1 diabetes

Abbreviations DIPP: Type I Diabetes Prediction and Prevention Project - GADA: Glutamic acid decarboxylase autoantibodies - HR: Hazard ratio - IAA: Insulin autoantibodies - IA-2A: Islet antigen 2 antibodies - ICA: Islet cell antibodies - JDFU: Juvenile Diabetes Foundation unit

\section{Introduction}

Genetic susceptibility to type 1 diabetes is defined by at least four gene loci. The HLA region is the major disease 
locus and the primary determinant is $H L A-D Q B 1$, a class II gene, the effect of which is profoundly modified by the adjacent $H L A-D Q A 1$ and $H L A-D R B 1$ genes and several other loci in the class I and class III regions [1-5]. In addition to the HLA region, the insulin gene (INS; IDDM2 locus), the CTLA4 region (cytotoxic T-lymphocyte-associated protein 4; IDDM12 locus) and the gene PTPN22 (lymphoid tyrosine phosphatase; 1p13, OMIM 600716) are confirmed to be associated with disease susceptibility [6-10].

The recent finding of association between the lymphoid protein tyrosine phosphatase (LYP) Arg620Trp variant (which corresponds to the PTPN22 C1858T polymorphism) and type 1 diabetes has been reproduced in several white populations [10-17]. In addition, this variant appears to be a common predisposing factor for other autoimmune diseases, such as rheumatoid arthritis, systemic lupus erythematosus, Graves' disease, Hashimoto thyroiditis and autoimmune Addison's disease [11, 14, 18-20]. LYP is expressed in lymphocytes and acts by binding intracellular kinases such as C-terminal Src tyrosine kinase (Csk). LYP seems to be directly involved in setting thresholds for T cell receptor signalling [21, 22]. The Arg620Trp functional polymorphism may be the causative disease variant, as Bottini et al. [10] showed that this amino acid substitution disrupts interaction with the tyrosine kinase, which could lead to pathogenic $\mathrm{T}$ cell responses and may be the defect that underlies the development of autoimmunity.

In type 1 diabetes, the initiation of the beta-cell-specific immune process often occurs early in life, and several years later it results in clinical disease in most individuals. Early histological and functional studies indicate that the disease is caused by $\mathrm{T}$ lymphocytes infiltrating the pancreatic islets $[23,24]$. The disease-specific immune events are, however, reflected in the appearance of beta-cell-specific autoantibodies, which are useful tools for the prediction of progression to clinical disease [25-27]. To understand the disease pathomechanism, it is crucial to explore the role of genetic factors in the emergence of beta cell autoimmunity. Previously, it has been shown that the DR4-DQ8 haplotype is associated with the appearance of insulin autoantibodies (IAA) and islet antigen-2 autoantibodies (IA-2A), whereas glutamic acid decarboxylase autoantibodies (GADA) develop more frequently in individuals carrying the $D R 3$ $D Q 2$ haplotype [28-31]. Recently, in a large Finnish birth cohort we observed a differential contribution of the $D R B 1^{*} 04$ alleles to the development of beta cell autoimmunity. Importantly, we also found that the INS locus plays a central role in the emergence of IAA and that the subsequent appearance of multiple autoantibodies is linked to IAA [32]. Our findings suggested that, in children who develop IAA, insulin autoimmunity may represent the primary event that is controlled, at least in part, by the INS locus.

In this study we examined the role of PTPN22 in the development of type 1 diabetes-associated autoimmunity and its contribution to the genetic control of progression to clinical disease. For this purpose we employed a large population-based cohort of infants who are prospectively followed from birth in the framework of the Type I Diabetes Prediction and Prevention Project (DIPP). This study population carries risk HLA genotypes and is well characterised in terms of clinical data and for genetic and immunological disease markers [33].

In addition, we explored the heterogeneity of the disease-predisposing effect of PTPN22 in relation to HLA genes, INS and CTLA4 genotypes, sex and age.

\section{Subjects and methods}

\section{Study design}

Firstly, the effect of the LYP Arg620Trp (PTPN22 C1858T; rs2476601) polymorphism on humoral beta cell autoimmunity and disease progression was evaluated in a population-based prospective follow-up cohort of young children with increased type 1 diabetes susceptibilitythe DIPP cohort. In the framework of the DIPP, screening for risk-associated HLA genotypes is performed in all infants born at three university hospitals in Finland: Turku, Tampere and Oulu [33]. Infants (about 7500 by May 2004) with risk $H L A-D Q B 1$ genotypes (DR3-DQ2/ $D R 4-D Q 8, D R 4-D Q 8 / X, D R 3-D Q 2 / Y$, where $X \neq D R 3$ $D Q 2, D Q B 1 * 0301, D Q B 1 * 0602$ and $Y \neq D R 7-D Q 2$, $D Q B 1 * 0301, * 0302, * 0602$ or $* 0603)$, were followed and sampled at intervals of 3-12 months, as described [33]. Islet cell antibodies (ICA) were analysed from all serum samples; if positive, IAA, GADA and IA-2A were tested in all samples available from that ICA-positive subject (Fig. 1).

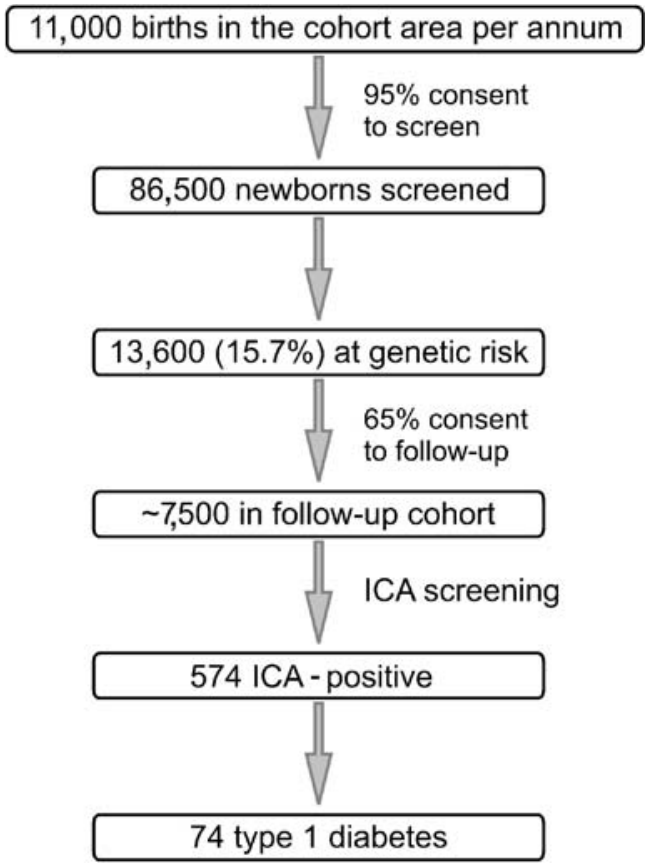

Fig. 1 Population screening and follow-up scheme in the Type I Diabetes Prediction and Prevention (DIPP) project (status in May, 2004). For the definition of the at-risk individuals, see Methods section in Subjects and methods 
Secondly, we explored the heterogeneity of the diseasepredisposing effect of PTPN22 in relation to HLA, the insulin gene, CTLA4 genotypes, sex and age at disease onset. To avoid population stratification effects we used both case-control and family-based designs.

The gene-gene interactions between PTPN22, HLA class II, INS and CTLA4 were explored first in stratified data sets and then tested for significance using regression analyses.

The WHO criteria were used for the diagnosis of type 1 diabetes [34].

The local ethics committees approved the study and informed consent was obtained from the parents of the participating subjects. The study was conducted according to the principles of the Declaration of Helsinki.

\section{Study populations}

\section{Antibody-positive children (DIPP cohort)}

All children who had developed ICA by May 2004 ( $n=574$, 180 with the $D R 3-D Q 2 / D R 4-D Q 8$ high-risk, 368 with the $D R 4-D Q 8 / X$ and 26 with the $D R 3-D Q 2 / Y$ moderate-risk genotypes; $53.5 \%$ boys) were tested for autoantibodies (IAA, GADA and IA-2A) and for genetic markers (PTPN22 C1858T, HLA-DRB1-DQA1-DQB1, INS -23 $H p h \mathrm{I}$ and CTLA4 CT60 polymorphisms). Two hundred and sixty-two infants developed biochemical autoantibodies, in addition to ICA, among whom 190 had multiple $(\geq 2)$ autoantibodies. At the time of this analysis the mean $( \pm \mathrm{SD})$ follow-up time in the DR3-DQ2/DR4-DQ8 and DR4-DQ8/ $X$ groups was $4.9 \pm 2.2$ years (range $0.5-9.3$ years), and somewhat shorter in those carrying the $D R 3-D Q 2 / Y$ combination (mean 3.9 \pm 1.4 years, range 0.5-6.1 years). By May 2004, 74 of these children (12.9\%) had progressed to clinical type 1 diabetes.

\section{Case-control series}

Samples from cases were collected from the Departments of Pediatrics at the Universities of Turku and Oulu ( $n=546$, $55.7 \%$ boys; mean age at diagnosis $8.2 \pm 4.1$ years). The control group comprised consecutive healthy infants born in the University Hospitals of Turku and Oulu ( $n=538$, $51.2 \%$ boys).

\section{Nuclear family series}

Families with one affected child were recruited mainly from the Departments of Pediatrics at the Universities of Turku and Oulu ( $n=245,52.9 \%$ boys; mean age at diagnosis $8.6 \pm 4.4$ years).

\section{Methods}

\section{Genotyping}

The PTPN22 C1858T (LYP Arg620Trp; rs2476601) polymorphism was genotyped with a homogeneous genotyping method [35]. The oligonucleotide sequences and reaction conditions are available upon request. Genotyping accuracy was evaluated by DNA sequencing 140 samples (MegaBace1000; GE Healthcare, Chalfont St Giles, UK). The genotypes obtained with the two methods were $100 \%$ identical. The methods for the $H L A-D Q B 1$ 'full house' and for $D Q A 1$ and $D R B 1^{*} 04$ typing have been described [36-38]. For the gene-gene interaction analysis in the case-control material, HLA genotypes were grouped among cases according to the presence or absence of the two major HLA risk haplotypes, as follows: HLA $D R 3$ DQ2/DR4-DQ8， DR4-DQ8/non-DR3-DQ2， DR3-DQ2/ non-DR4-DQ8, non- $D R 4-D Q 8 /$ non- $D R 3-D Q 2$. In the ICA-positive DIPP cohort, the heterogeneity of PTPN22 effects was tested between HLA genotype groups defined according to the screening classification criteria: $D R 3$ $D Q 2 / D R 4-D Q 8, D R 4-D Q 8 / X$ and $D R 3-D Q 2 / Y$. The INS -23 HphI polymorphism was typed as described previously $[32,36,39]$. The CTLA4 CT60 polymorphism was genotyped using minisequencing (SnuPe Kit, MegaBace 1000; GE Healthcare).

\section{Autoantibody assays}

The antibody assay parameters used have been described in detail [40]. The detection limit for ICA was 2.5 Juvenile Diabetes Foundation units (JDFU; sensitivity 100\%, specificity 98\%). IAA levels were quantified with a microassay [41, 42]. GADA and IA-2A were measured using specific radioligand assays [43, 44]. The cut-off values for IAA, GADA and IA-2A positivity were 1.56 , 5.36 and $0.43 \mathrm{RU}$ (relative units), respectively, being the 99th percentiles in a series comprising more than 370 nondiabetic Finnish children and adolescents. The disease sensitivities of the IAA, GADA and IA-2A assays were 44, 82 and $62 \%$, respectively, and the specificities were 98,98 and $100 \%$, respectively, based on the 2002 Diabetes Autoantibody Standardization Program Workshop.

\section{Statistical analysis}

Kaplan-Meier survival analysis was applied to evaluate genetic effects on the appearance of various autoantibodies. Differences between the survival curves were tested with the log rank test. Cox regression was employed to analyse the effects of various factors (antibody status, genotypes) on autoantibody-free survival. Disease associations of gene variants were evaluated by forward stepwise logistic regression analysis and, in some comparisons, using the $\chi^{2}$ 
statistic with Yates' correction. All statistical analyses were performed using SPSS for Windows (version 11.0.1; SPSS, Chicago, IL, USA). The transmission disequilibrium test was performed using Unphased software [45]. Values of $p$ lower than 0.05 were considered statistically significant.

\section{Results}

The effect of the PTPN22 C1858T variant on the emergence of beta-cell-specific autoimmunity and progression to clinical type 1 diabetes in the DIPP cohort

Figure 2 shows the Kaplan-Meier survival analysis of progression to type 1 diabetes in the ICA-positive DIPP follow-up cohort. Clinical disease appeared at a higher rate in children with the TT and TC genotypes than in those carrying the $\mathrm{CC}$ variant (hazard ratio $[\mathrm{HR}]=5.5,95 \% \mathrm{CI}$ 2.3-13.2 and $\mathrm{HR}=1.6$, 95\% CI 1.0-2.7, respectively; $p=0.0001)$. Using logistic regression analysis, we observed that the PTPN22 C1858T variant showed association with progression of autoimmunity reflected by the number of autoantibodies appearing during follow-up. Children carrying the TT and CT genotypes had an increased risk of developing additional autoantibodies (HRs for developing

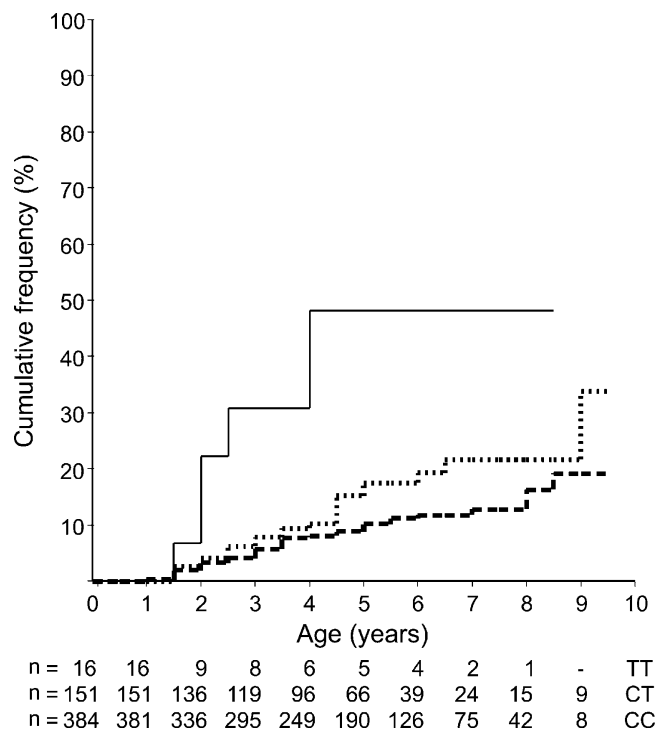

Fig. 2 The effect of $P T P N 22$ on progression to clinical type 1 diabetes in children with risk HLA genotypes. Cumulative disease frequencies are shown for different PTPN22 C1858T genotypes. Median disease-free survival was shorter in individuals carrying the TT genotype (5.6 years; 95\% CI 3.9-7.3 years; $n=16$ ) than in those with the TC or CC variant (8.0 years, $95 \%$ CI $7.6-8.6 ; n=151$ and 8.4 years, 95\% CI 8.2-8.7; $n=384$, respectively, $p=0.0001$ ). All children carried the HLA $D R 3-D Q 2 / D R 4-D Q 8$ or $D R 4-D Q 8 / X$ combination, in which $X \neq D R 3-D Q 2, D Q B 1^{*} 0301$ or $D Q B 1^{*} 0602$. Solid line subjects with the TT genotype; dotted line subjects carrying the CT combination; dashed line subjects with the $\mathrm{CC}$ variant an additional antibody were 4.1 [95\% CI 1.5-11.6] and 1.64 [ $95 \%$ CI 1.1-2.4], respectively; $p=0.003$ ).

ICA

The effect of the PTPN22 variant on the emergence of ICA was estimated by comparing the genotype frequencies of the children with ICA only (TT $1.3 \%$, CT $24.1 \%$, CC $74.6 \%$ ) with those seen in healthy controls (see data in Table 3). No difference was observed.

\section{IAA, GADA and IA-2A}

Genetic effects on the appearance of these autoantibodies were estimated by survival analysis. The cumulative frequencies of IAA were higher and antibody-free survival was shorter among children with the TT genotype $(76.0 \%$, 3.0 years, $95 \%$ CI $1.3-4.6$ years) compared with those with the genotypes CT ( $46.5 \%, 5.9$ years, 95\% CI 5.3-6.6 years) and CC $(42.0 \%, 6.5$ years, 95\% CI $6.2-6.9 ; p=0.00007)$. The hazard ratio for the emergence of IAA in subjects with the TT combination was 3.7 (95\% CI 2.0-6.9) and was 1.3 (95\% CI 1.0-1.8) in those with the CT genotype. The effect of the $\mathrm{T}$ allele on the emergence of IAA was strongly significant also when the study group was stratified according to the presence of IA-2A and GADA (adjusted $p=0.0001$; Table 1). Accordingly, in Cox regression the effect of the PTPN22 TT variant was significant after adjusting for GADA and IA-2A carrier status (adjusted HR for the appearance of IAA in the C1858T TT group was $3.2,95 \%$ CI 1.7-5.9; reference genotype, CC, $p=0.001$ ).

When the effect of the PTPN22 on the appearance of GADA was analysed, the TT variant appeared to be associated with a higher cumulative frequency of GADA; however, when adjusted for IAA status, the effect was not significant (Table $1 ; p=0.073$ ). Similarly, the emergence of IA-2A was not significantly influenced by the PTPN22 genotype after adjusting for IAA status (adjusted $p=0.17$ ).

The effect of the PTPN22 was also analysed in relation to which autoantibody reactivity appeared first during the follow-up (Fig. 3). The 1858TT genotype was associated with higher cumulative IAA frequency and shorter IAAfree survival than the $\mathrm{CC}$ variant (median IAA-free survival with TT genotype was 3.2 years, 95\% CI $1.5-$ 5.0 versus 7.0 years, $95 \%$ CI $6.7-7.4$ years in the group with CC combination; log rank $21.4, p=0.00008, d f=2$; Fig. 3a). However, no effect of PTPN22 was seen on the emergence of GADA ( $p=0.27$; Fig. $3 \mathrm{~b})$ or IA- $2 \mathrm{~A}$ ( $p=0.57$; Fig. 3c).

We also evaluated how PTPN22 influenced the transient appearance of autoantibodies (ICA $n=84,14.6 \%$; IAA $n=77,13.4 \%$; GADA $n=37,6.4 \%$; IA-2A $n=7,1.2 \%$ ). No significant effects were found, although, an increase in the cumulative frequency of transient IAA was suspected in subjects with the $1858 \mathrm{TT}$ genotype $(p=0.11)$. 
Table 1 Survival analysis of the effect of the PTPN22 C1858T polymorphism on the appearance of autoantibodies in the ICA-positive DIPP cohort

\begin{tabular}{|c|c|c|c|c|c|}
\hline $\begin{array}{l}\text { Autoantibody } \\
\text { analysed }\end{array}$ & Study subjects & $\begin{array}{l}P T P N 22 \mathrm{C} 1858 \mathrm{~T} \\
\text { genotype }(n)\end{array}$ & $\begin{array}{l}\text { Cumulative frequencies } \\
\text { of autoantibodies }(\%)\end{array}$ & $\begin{array}{l}\text { Antibody-free survival } \\
\text { (years): } \text { mean }^{\text {a }}(95 \% \mathrm{CI})\end{array}$ & $\begin{array}{l}P T P N 22 \text { effect: } \\
\text { adjusted } p \text { value }^{\mathrm{b}}\end{array}$ \\
\hline \multirow[t]{6}{*}{ IAA } & \multirow[t]{3}{*}{ GADA/IA-2A-positive } & TT (9) & 100.0 & $1.2(1.0-1.4)$ & \multirow[t]{6}{*}{0.0001} \\
\hline & & TC (72) & 83.5 & $3.2(2.6-4.0)$ & \\
\hline & & CC (131) & 78.2 & $3.5(2.9-3.8)$ & \\
\hline & \multirow[t]{3}{*}{ GADA/IA-2A-negative } & TT (6) & 37.5 & $5.8(2.9-8.7)$ & \\
\hline & & $\mathrm{TC}(80)$ & 16.6 & $8.2(7.7-8.8)$ & \\
\hline & & $\mathrm{CC}(254)$ & 10.1 & $8.3(8.0-9.0)$ & \\
\hline \multirow[t]{6}{*}{ GADA } & \multirow[t]{3}{*}{ IAA-positive } & TT (11) & 84.4 & $2.0(1.4-2.7)$ & \multirow[t]{6}{*}{0.073} \\
\hline & & $\mathrm{TC}(64)$ & 89.0 & $3.0(2.4-3.6)$ & \\
\hline & & CC (132) & 76.0 & $4.1(3.5-4.7)$ & \\
\hline & \multirow[t]{3}{*}{ IAA-negative } & TT (4) & 0 & - & \\
\hline & & $\mathrm{TC}(87)$ & 27.4 & $7.9(7.4-8.5)$ & \\
\hline & & CC (253) & 14.2 & $8.6(8.3-8.8)$ & \\
\hline \multirow[t]{6}{*}{ IA-2A } & \multirow[t]{3}{*}{ IAA positives } & TT (11) & 87.5 & $2.2(1.6-2.7)$ & \multirow[t]{6}{*}{0.17} \\
\hline & & TC (64) & 86.1 & $3.4(2.7-4.1)$ & \\
\hline & & CC (132) & 80.0 & $4.3(3.7-4.8)$ & \\
\hline & \multirow[t]{3}{*}{ IAA negatives } & TT (4) & 0 & - & \\
\hline & & TC (87) & 21.4 & $8.5(8.2-8.9)$ & \\
\hline & & CC (253) & 13.9 & $8.8(8.5-9.0)$ & \\
\hline
\end{tabular}

The number of children in each genotype category is shown in parentheses

${ }^{a}$ Median values for antibody-free survival could not be calculated in some cases because the cumulative antibody frequencies were lower than $50 \%$; therefore, the mean values are reported

${ }^{\mathrm{b}} \log$ rank test; $d f=2$ for all $p$ values

Heterogeneity in the effect of PTPN22

on the emergence of IAA according to HLA class II, INS, CTLA4, age and sex

The appearance of IAA in the three PTPN22 genotype groups stratified according to HLA class II, INS and CTLA4 genotypes is shown in Table 2. IAA appeared at a higher rate in children carrying the TT genotype both in the $D R 3-D Q 2 / D R 4-D Q 8$ and in the $D R 4-D Q 8 / X$ risk category (adjusted $p=0.0003$ ). In addition, Cox regression revealed a higher risk of developing IAA in the $D R 3-D Q 2 / D R 4-D Q 8$ category in all PTPN22 genotype groups compared with the $D R 4-D Q 8 / X$ group (HR=1.5, 95\% CI 1.1-2.1; $p=0.015$ ). The emergence of IAA could not be estimated reliably among boys with the $D R 3-D Q 2 / Y$ genotype because of the small numbers.

We analysed the effect of PTPN22 on the development of IAA in data sets stratified according to the DRBI subtype on the $D R 4-D Q 8$ haplotypes. We observed that the effect of PTPN22 was present both in the $D R B 1^{*} 0401$ and *0404 groups (adjusted $p=0.0002$ ), with a higher cumulative IAA frequency in all PTPN22 categories in the $D R B 1^{*} 0401$ group $(p=0.00004)$. Only one child developed IAA in the $D R B 1^{*} 0403$ group.

The strong effect of PTPN22 on the emergence of IAA was present both in the INS (-23) HphI AA and AT/
TT INS genotype groups. In addition, IAA appeared at a higher rate in the INS (-23) HphI AA group (HR=2.1, 95\% CI $1.5-3.0 ; p=0.00007)$.

The PTPN22 C1858T polymorphism affected the appearance of IAA in all three CTLA4 CT60 genotype groups in a similar way $(p=0.0005)$, and we did not observe any interaction between PTPN22 and CTLA4 in this respect ( $p=0.53$ ).

Interestingly, sex influenced the appearance of IAA, as boys carrying the PTPN22 1858TT genotype had a higher risk of developing IAA than girls $(\mathrm{HR}=1.4,95 \%$ CI $1.1-1.8 ; p=0.033$ ).

Additionally, we observed that at the time of IAA seroconversion children with the TT genotype were younger (2.4 \pm 0.7 years) than those with $\mathrm{CT}$ or $\mathrm{CC}$ genotype (3.8 \pm 0.2 and $4.1 \pm 0.1$ years, respectively; $p=0.016$ ). No age-dependent heterogeneity in the PTPN22 genotype distribution was detected in the DIPP cohort $(p=0.37)$.

Using Cox regression analysis, we estimated the effect of the PTPN22 variant on the emergence of IAA adjusted for the effects of HLA, INS, CTLA4 and sex. Individuals with the TT and CT genotypes had a strongly increased probability of developing IAA (adjusted $\mathrm{HR}=4.6,95 \%$ CI 2.4-9.0; and $\mathrm{HR}=1.5,95 \%$ CI 1.5-3.0, respectively; $p=0.000013$ ). 

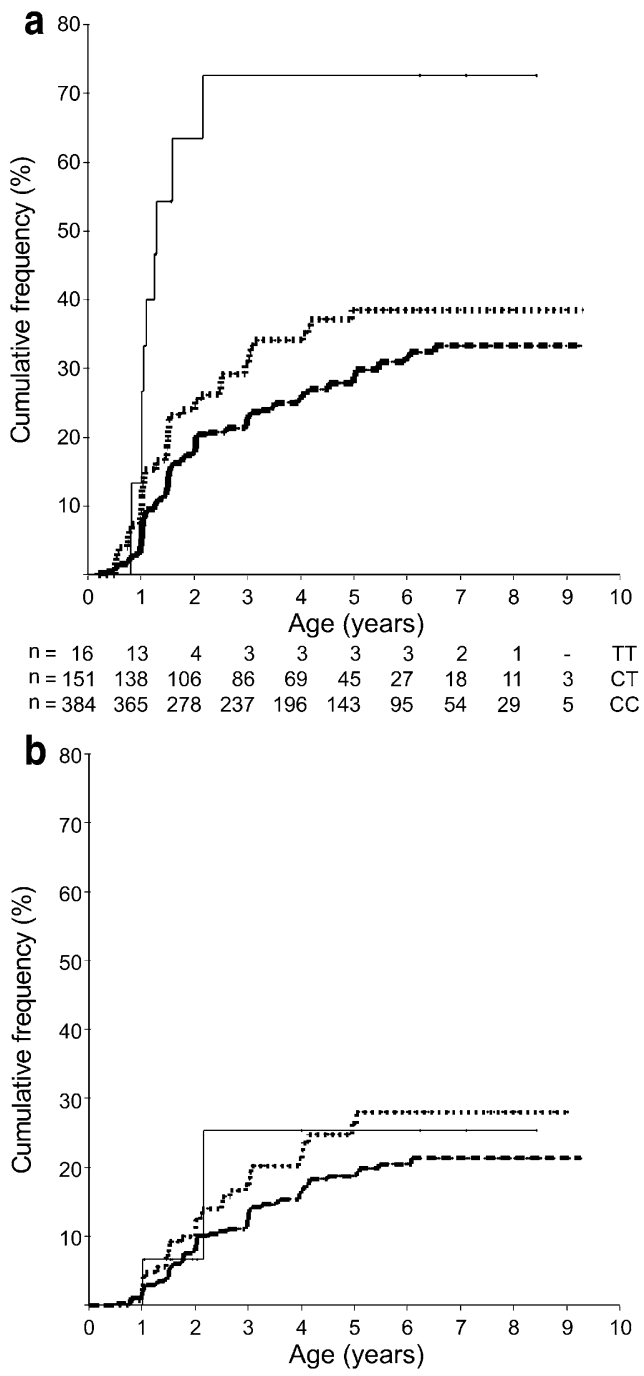
$\begin{array}{rrrrrrrrrrr}\mathrm{n}=16 & 15 & 6 & 4 & 4 & 3 & 3 & 2 & 1 & - & \text { TT } \\ \mathrm{n}=151 & 145 & 113 & 93 & 71 & 46 & 29 & 20 & 11 & 2 & \mathrm{CT}\end{array}$ $\begin{array}{rrrrrrrrrrr}\mathrm{n}=384 & 374 & 297 & 246 & 204 & 153 & 100 & 63 & 32 & 6 & \mathrm{CC}\end{array}$

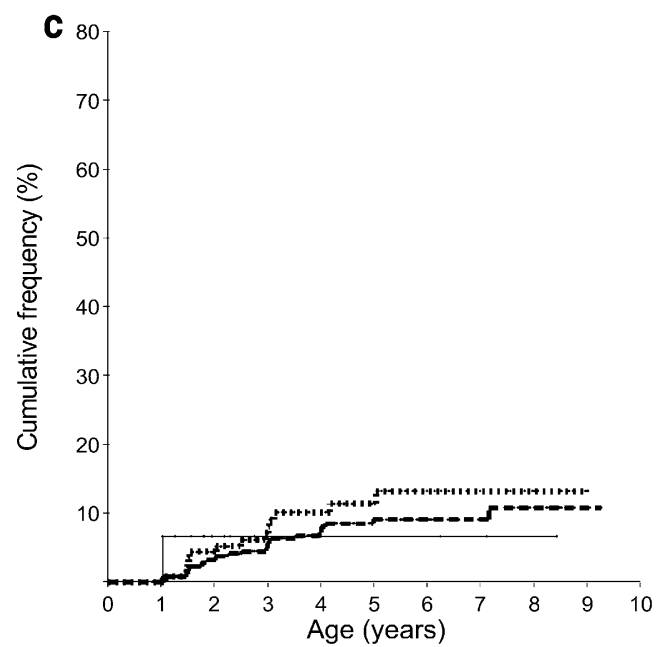

$\begin{array}{lllllllllll}n=16 & 15 & 8 & 4 & 4 & 3 & 3 & 2 & 1 & -T T\end{array}$ $\begin{array}{rrrrrrrrrrr}\mathrm{n}=151 & 151 & 116 & 96 & 74 & 52 & 30 & 20 & 13 & 2 & \mathrm{CT}\end{array}$ $\mathrm{n}=384 \quad \begin{array}{llllllllll}379 & 313 & 255 & 212 & 153 & 103 & 63 & 34 & 6 & \mathrm{CC}\end{array}$
Fig. 3 The effect of PTPN22 on the appearance of IAA, GADA and IA-2A as first autoantibody during follow-up. a Appearance of IAA as the first autoantibody during follow-up in children carrying different PTPN22 C1858T genotypes. Cumulative frequency of IAA was higher in the TT and CT groups than in children with the CC genotype ( 73.6 and 38.7 vs $33.5 \%$; $\log$ rank $21.4, d f=2, p=0.00008$; $n=168$ ). b Emergence of GADA as the first autoantibody in subjects carrying different PTPN22 C1858T genotypes $(p=0.27 ; n=96)$. c Development of IA-2A as the first autoantibody in different PTPN22 C1858T genotype groups $(p=0.57 ; n=42)$. Solid lines subjects with the TT genotype; dotted lines subjects carrying the CT combination; dashed lines subjects with the CC variant

Heterogeneity of the effect of PTPN22 on type 1 diabetes susceptibility

In the case-control series, the disease association of $P T P N 22$ and its interaction with HLA DR-DQ, CTLA4, $I N S$, sex and age at disease onset were analysed using forward stepwise logistic regression analysis (Table 3). As expected, the PTPN22 C1858T polymorphism showed a strong disease association $\left(p=2.1 \times 10^{-8}\right)$. The odds ratios (OR) for the TT and CT genotypes were 3.1 (95\% CI 1.4 $5.2)$ and $2.9(95 \%$ CI $2.0-4.2)$, respectively. The frequency of the $\mathrm{T}$ allele was $23.9 \%$ in cases and $13.9 \%$ in controls $\left(p=8 \times 10^{-6}\right)$. In the cases, the exploratory analysis of the distribution of $P T P N 22$ C1858T genotypes stratified according to HLA-DQB1, CTLA4 CT60 and INS (-23 $H p h \mathrm{I})$ genotypes, age at disease presentation and sex indicated heterogeneity for HLA, INS and sex. The significance of these interactions was estimated using a case-only regression model (Table 3). A significant effect of sex on PTPN22-encoded disease susceptibility was observed, as affected boys with the TT genotype had higher disease risk than girls (OR 1.5, 95\% CI 1.1-2.2; $p=0.021$ ). We found no significant effect of the age at diagnosis, although the TT genotype appeared to be more prevalent among patients at a young age. However, we detected heterogeneity in the disease-predisposing effect of PTPN22 among different HLA class II genotypes, as the 1858TT and CT genotypes were found at higher frequencies in patients carrying the HLA $D R 3-D Q 2 /$ non- $D R 4-D Q 8$ and non- $D R 3-D Q 2 /$ non- $D R 4-D Q 8$ combinations compared with those with the $D R 3-D Q 2 / D R 4-D Q 8$ or $D R 4-D Q 8 /$ non- $D R 3-D Q 2$ variants $(p=0.0004)$. The data also suggested that the effect of PTPN22 is stronger in children who carry INS (-23) HphI AT or TT compared with the AA group $(p=0.04)$. No significant heterogeneity in the effect of PTPN22 was observed in relation to CTLA4.

In the controls, no heterogeneity was observed in the distribution of PTPN22 genotypes, when stratified according to factors listed in Table 3.

In the nuclear family series, the $\mathrm{T}$ allele was preferentially transmitted to affected offspring (transmission disequilibrium test; 109 transmitted, $61.6 \% ; p=0.001)$. The transmission rate to affected boys was higher $(67.1 \%$; $p=0.0004)$ than that to girls $(55.3 \% ; p=0.317)$. This difference was valid both for paternal and maternal transmission (to boys, 70.2 and $65.3 \%$; to girls, 50.83 and $55.6 \%$, respectively). In the nuclear family series, the parental $\mathrm{T}$ allele frequency was $17.6 \%$. There was no 
Table 2 Survival analysis of the effect of the PTPN22 C1858T polymorphism on the appearance of insulin autoantibodies in ICA-positive children carrying various $H L A-D Q B 1, I N S$ and CTLA4 genotypes

\begin{tabular}{|c|c|c|c|c|}
\hline & $\begin{array}{l}P T P N 22 \mathrm{C} 1858 \mathrm{~T} \\
\text { genotype }(n)\end{array}$ & $\begin{array}{l}\text { Cumulative IAA } \\
\text { frequency }(\%)\end{array}$ & $\begin{array}{l}\text { IAA-free survival in } \\
\text { years: mean }{ }^{\mathrm{a}}(95 \% \mathrm{CI})\end{array}$ & $\begin{array}{l}P T P N 22 \text { effect: } \\
\text { adjusted } p \text { value }{ }^{\mathrm{b}}\end{array}$ \\
\hline \multirow[t]{3}{*}{$D R 3-D Q 2 / D R 4-D Q 8^{\mathrm{c}}$} & TT (5) & 100.0 & $1.3(0.8-1.7)$ & 0.0003 \\
\hline & $\mathrm{TC}(49)$ & 52.9 & $5.3(4.2-6.4)$ & \\
\hline & CC (120) & 52.5 & $5.9(5.3-6.6)$ & \\
\hline \multirow[t]{3}{*}{$D R 4-D Q 8 / X^{\mathrm{d}}$} & TT (10) & 62.5 & $3.9(1.6-6.2)$ & \\
\hline & $\mathrm{TC}(95)$ & 45.2 & $6.0(5.2-6.7)$ & \\
\hline & CC (250) & 36.9 & $6.8(6.3-7.3)$ & \\
\hline \multirow[t]{3}{*}{$D R B 1^{*} 0401^{\mathrm{e}}$} & TT (9) & 100 & $1.3(0.9-1.52)$ & 0.0002 \\
\hline & CT (96) & 60.9 & $4.7(4.0-5.5)$ & \\
\hline & CC (241) & 46.1 & $6.1(5.6-6.6)$ & \\
\hline \multirow[t]{3}{*}{$D R B 1 * 0403$} & TT (0) & - & - & \\
\hline & CT (9) & 0 & - & \\
\hline & CC (13) & 11.1 & $7.9(6.8-9.0)$ & \\
\hline \multirow[t]{3}{*}{$D R B 1 * 0404$} & TT (4) & 0 & - & \\
\hline & CT (36) & 29.5 & $7.3(6.2-8.3)$ & \\
\hline & CC (106) & 41.6 & $6.9(6.2-7.5)$ & \\
\hline \multirow[t]{3}{*}{$I N S(-23) H p h \mathrm{I} \mathrm{AA}^{\mathrm{f}}$} & TT (9) & 77.8 & $2.9(0.9-4.8)$ & 0.0002 \\
\hline & $\mathrm{TC}(92)$ & 51.7 & $5.4(4.6-6.2)$ & \\
\hline & CC (258) & 47.7 & $6.1(5.7-6.6)$ & \\
\hline \multirow[t]{3}{*}{ INS (-23) HphI AT and TT } & TT (4) & 75.0 & $1.2(1.0-1.4)$ & \\
\hline & $\mathrm{TC}(49)$ & 34.8 & $6.8(5.8-7.9)$ & \\
\hline & CC (108) & 24.4 & $7.6(7.1-8.2)$ & \\
\hline \multirow[t]{3}{*}{$C T L A 4$ CT60 AA ${ }^{\mathrm{g}}$} & TT (6) & 50.0 & $4.8(1.8-7.7)$ & 0.0005 \\
\hline & $\mathrm{TC}(28)$ & 59.1 & $5.4(4.0-6.7)$ & \\
\hline & $\mathrm{CC}(83)$ & 41.3 & $6.7(5.9-7.4)$ & \\
\hline \multirow[t]{3}{*}{ CTLA4 CT60 AG } & TT (4) & 75.0 & $2.8(0.4-5.3)$ & \\
\hline & $\mathrm{TC}(81)$ & 45.0 & $5.7(4.9-6.6)$ & \\
\hline & CC (182) & 44.8 & $6.2(5.7-6.8)$ & \\
\hline \multirow[t]{3}{*}{ CTLA4 CT60 GG } & TT (5) & 100.0 & $1.2(0.9-1.4)$ & \\
\hline & $\mathrm{TC}(43)$ & 40.6 & $5.7(4.7-6.6)$ & \\
\hline & CC (121) & 40.0 & $6.6(6.0-7.3)$ & \\
\hline
\end{tabular}

The number of children in each genotype category is shown in parentheses

${ }^{a}$ Median values for the antibody-free survival could not be calculated in some cases because the cumulative antibody frequencies were lower than $50 \%$; therefore, the mean values are reported

${ }^{\mathrm{b}} \mathrm{Log}$ rank test; $d f=2$ for all $p$ values

${ }^{c} p=0.015$ vs $D R 4-D Q 8 / X(d f=1)$

${ }^{\mathrm{d}} X \neq D R 3-D Q 2, D Q B 1 * 0301, D Q B 1 * 0602$

e $p=0.00004$ vs $D R B 1^{*} 0403$ and $D R B 1^{*} 0404(d f=2)$

All $D R B 1 * 04$ subtypes carry $D Q 8$

${ }^{\mathrm{f}} p=0.00007$ vs $I N S(-23) H p h \mathrm{I}$ AT and TT $(d f=1)$

${ }^{\mathrm{g}} p=0.53$ vs CTLA4 CT60 AG and GG $(d f=2)$

difference in parental $\mathrm{T}$ allele frequency between families with affected boys and girls.

\section{Discussion}

This study revealed several important novel findings on the effect of the PTPN22 C1858T (LYP Arg620Trp) polymorphism on the development of humoral beta cell autoimmunity and disease progression.
Firstly, we observed that the PTPN22 C1858T variant was strongly associated with progression of beta-cellspecific autoimmunity, as ICA-positive children carrying the TT genotype had a four-fold higher risk of developing an additional molecular autoantibody than those with the CC genotype. This implies that the PTPN22 1858TT genotype defines a more homogeneous high-risk population within the given HLA risk group, which is more suitable for future prevention trials than those identified by the genetic screening criteria used so far. 
Table 3 Analysis of heterogeneity in the type 1 diabetes predisposing effect of PTPN22 in the Finnish population

\begin{tabular}{|c|c|c|c|c|}
\hline \multirow[t]{2}{*}{ Subjects } & \multicolumn{3}{|c|}{ PTPN22 C1858T genotype $n(\%)$} & \multirow[t]{2}{*}{$p$ value } \\
\hline & TT & $\mathrm{CT}$ & $\mathrm{CC}$ & \\
\hline All cases & $30(5.5)$ & $200(36.6)$ & $316(57.9)$ & 0.000000021 \\
\hline Male & $17(5.9)$ & $118(40.8)$ & $154(53.3)$ & 0.021 \\
\hline Female & $13(5.1)$ & $82(31.9)$ & $162(63.0)$ & \\
\hline \multicolumn{5}{|l|}{ Age at diagnosis } \\
\hline $0-4.99$ years & $9(7.6)$ & $48(40.3)$ & $62(52.1)$ & 0.305 \\
\hline 5-9.99 years & $11(6.3)$ & $64(36.8)$ & $99(56.9)$ & \\
\hline 10-14.99 years & $9(4.0)$ & $82(36.8)$ & $132(59.2)$ & \\
\hline HLA $D R 3-D Q 2 / D R 4-D Q 8$ & $3(3.1)$ & $33(33.7)$ & $62(63.3)$ & 0.0004 \\
\hline HLA $D R 4-D Q 8 /$ non- $D R 3-D Q 2$ & $16(5.4)$ & $97(33.0)$ & $181(61.6)$ & \\
\hline HLA $D R 3-D Q 2 /$ non- $D R 4-D Q 8$ & $7(7.3)$ & $45(46.9)$ & $44(45.8)$ & \\
\hline HLA non- $D R 3-D Q 2 /$ non- $D R 4-D Q 8$ & $4(6.9)$ & $25(43.1)$ & $29(50.0)$ & \\
\hline INS (-23) HphI AA & $21(5.3)$ & $142(36.1)$ & $230(58.5)$ & 0.040 \\
\hline INS (-23) HphI AT and TT & $8(7.4)$ & $45(41.7)$ & $55(50.9)$ & \\
\hline CTLA4 CT60 AA & $6(13.3)$ & $16(35.6)$ & $23(51.1)$ & 0.095 \\
\hline CTLA4 CT60 AG & $7(3.5)$ & $76(38.4)$ & $115(58.1)$ & \\
\hline CTLA4 CT60 GG & $15(5.6)$ & $94(35.1)$ & $159(59.3)$ & \\
\hline Controls & $14(2.6)$ & $122(22.7)$ & $402(74.7)$ & \\
\hline
\end{tabular}

The global effect of PTPN22 was estimated using a logistic regression model comprising both cases and controls Effects of sex, age at diagnosis, HLA, INS and CTLA4 were estimated with a case-only regression model

Importantly, the emergence of IAA showed a strong association with the PTPN22 TT genotype, which underlines the significance of this variant in the regulation of beta-cell-specific autoimmunity. In contrast, the development of GADA and IA-2A was not significantly influenced by this polymorphism. It should be noted that the analysis of genetic effects on various autoantibodies was complicated by the fact that most $(81.7 \%)$ of the IA-2A- and GADA-positive children also tested positive for IAA. However, the Cox regression that enabled us to adjust for the presence of other autoantibodies, and also the analysis of the autoantibody reactivity that appeared first during follow-up, indicated that the PTPN22 TT variant was primarily associated with the emergence of IAA (Fig. 3). Notably, individuals with the TT genotype had a considerably higher probability of developing IAA or other additional autoantibodies than those with the CT heterozygous genotype, which is consistent with a possible gene dose effect.

The effect of PTPN22 on the appearance of IAA was more pronounced in the high-risk HLA $D R 3-D Q 2 / D R 4-$ $D Q 8$ genotype group than in the medium-risk $D R 4-D Q 8 / X$ category, which could be explained by the higher number of disease-predisposing HLA heterodimer molecules, which promotes the autoimmune process in the former individuals. We also observed another HLA class II effect on PTPN22-encoded beta-cell-specific autoimmunity. The
$D R B 1^{*} 04$ allele present in the DR4-DQ8 HLA haplotype influenced the emergence of IAA in children with the TT and CT PTPN22 genotypes, as those carrying the $D R B 1 * 0401$ variant had a higher probability of developing this autoantibody compared with subjects with the $D R B 1 * 0404$ and $* 0403$ alleles. This effect could be caused by differences in the peptide binding specificity of the encoded DR molecule. The differential effect of $D R B 1 * 04$ alleles on the emergence of IAA is probably related to the different degrees of type 1 diabetes risk they confer [2, 46].

We have reported previously an association of IAA with the INS locus in the same cohort [32], and similar observations were made in two other studies on patients with newly diagnosed type 1 diabetes and on first-degree relatives [30, 47]. Therefore, we stratified the data set according to the INS genotype and observed that the effect of PTPN22 was present in both INS risk categories. In addition, the appearance of IAA was more frequent in children in the INS $(-23) H p h$ I AA risk group in all PTPN22 genotype categories, which implicates an additive effect of these two loci on the emergence of IAA. Our data also suggest not only that PTPN22 is associated with the emergence of IAA, but also that it seems to accelerate insulin-specific autoimmunity, as children with the 1858TT genotype developed IAA at an earlier age than others.

We found no evidence of heterogeneity in the effect of PTPN22 on the appearance of IAA in relation to the CTLA4 
genotype. This is in accordance with previous observations from us and from other groups indicating a lack of association of CTLA4 with the emergence of diabetesspecific autoantibodies [30, 48].

Previously, we proposed the existence of two different pathways that lead to the emergence of beta cell autoimmunity [32]. In the INS-dependent pathway, insulin autoimmunity seems to play a central role in the initiation of the disease process, whereas in IAA-negative individuals the initiation of beta cell autoimmunity and the development of GADA and IA-2A are probably controlled by factors other than the INS locus. Data from the present study indicate that the PTPN22 C1858T polymorphism, which corresponds to the LYP Arg620Trp variant, may be primarily involved in the INS-dependent pathway by modulating insulin-specific humoral autoimmunity. Since PTPN22 is associated with other autoimmune diseases in addition to type 1 diabetes, such as Graves' disease and rheumatoid arthritis $[11,14,19]$, it is likely that the LYP protein is involved in antigen-driven, disease-specific immune phenomena. Accordingly, our data suggest that insulin could be a target antigen for the action of PTPN22 in type 1 diabetes.

We also observed in this study that the appearance of ICA alone was not associated with PTPN22. Most of the children who developed ICA had an antibody titre below 20 JDFU (median 5.0 JDFU; interquartile range 4.0-8.0 JDFU), which is much lower than the ICA titre in those who had additional autoantibodies (44 JDFU, 8.0-219.0 JDFU; $\left.p<10^{-6}\right)$. This observation is in accordance with our recent findings implicating that the emergence of low-titre ICA alone is not related to the diabetes-specific autoimmune process [32].

The analysis of the heterogeneity of the effect of PTPN22 on type 1 diabetes susceptibility corroborated previous observations that the PTPN22 1858T allele is associated with type 1 diabetes [10-12, 14-16]. However, unlike in other studies, in both the case-control and the family series we observed that boys carrying the $T$ allele were at higher risk of disease than girls. This sex-specific bias in the action of PTPN22 was underlined by the findings in the antibody-positive cohort, which indicated that males carrying the TT genotype had a higher risk of developing IAA than females. In addition, the effect of PTPN22 appeared to be stronger in individuals carrying non-DR4-DQ8 genotypes. Such findings have not been reported before and suggest a particular molecular mechanism that may play a more important role in males, and involves PTPN22 and HLA DR3-DQ2 or neutral HLA haplotypes. A similar sex-specific HLA effect was implicated by our data in Finns [37, 49] and also by Cucca et al. [50], indicating that males with the DR3-DQ2 haplotype have a higher disease risk than girls. The regression analysis also suggested heterogeneity in the effect of PTPN22 on type 1 diabetes susceptibility in children with different $I N S$ genotypes, while no interaction between PTPN22 and CTLA4 was detected. Since the regression analysis in the case-control series had limited statistical power to detect gene-gene interaction phenomena between loci with such weak $(\mathrm{OR}<2)$ disease association, these findings require replication in larger series.

In conclusion, the present study provides strong evidence that the PTPN22 C1858T polymorphism regulates diabetes-specific autoimmunity and is a marker of disease progression. In addition, it appears to be primarily associated with insulin-specific humoral autoimmunity, indicating an underlying mechanism for the LYP 620Trp variant protein. Importantly, this polymorphism seems to have an additive effect with the INS locus on the emergence of IAA. We also observed a complex interaction pattern of PTPN22 with certain HLA class II genotype combinations and with sex. It should be noted that in the present analysis all children in the autoantibody positive cohort were ICApositive; therefore, the role of the PTPN22 C1858T variant on the emergence of humoral beta cell autoimmunity in ICA-negative subjects needs further investigation. Further functional studies are also required to refine our knowledge on the multifaceted molecular mechanisms by which PTPN22 contributes to the development of type 1 diabetes.

Acknowledgements This study was supported by the Juvenile Diabetes Research Foundation (JDRF grants 4-1998-274 and 41999-731), the JDRF/European Foundation for the Study of Diabetes/Novo Nordisk Focused Research Grant for Type 1 Diabetes, Novo Nordisk Foundation, Turku, Oulu and Tampere University Hospitals and the Academy of Finland. We thank all the personnel of the DIPP, particularly E. Nirhamo, P. Nurmi, J. Mantere, M. Karlson, R. Suominen, T. Laakso, S. Anttila, S. Heikkilä, R. Päkkilä and P. Salmijärvi for their skilful technical assistance. We wish to thank $\mathrm{T}$. Vahlberg for valuable help with the statistics. We are most grateful to all the children who participated in the DIPP and their families for their contribution.

\section{References}

1. Pociot F, McDermott MF (2002) Genetics of type 1 diabetes mellitus. Genes Immun 3:235-249

2. Hermann R, Turpeinen H, Laine AP et al (2003) HLA DR-DQencoded genetic determinants of childhood-onset type 1 diabetes in Finland: an analysis of 622 nuclear families. Tissue Antigens 62:162-169

3. Nejentsev S, Gombos Z, Laine AP et al (2000) Non-class II HLA gene associated with type 1 diabetes maps to the $240-\mathrm{kb}$ region near HLA-B. Diabetes 49:2217-2221

4. Noble JA, Valdes AM, Bugawan TL, Apple RJ, Thomson G, Erlich HA (2002) The HLA class I A locus affects susceptibility to type 1 diabetes. Hum Immunol 63:657-664

5. Zavattari P, Lampis R, Motzo C et al (2001) Conditional linkage disequilibrium analysis of a complex disease superlocus, IDDM1 in the HLA region, reveals the presence of independent modifying gene effects influencing the type 1 diabetes risk encoded by the major HLA-DQB1, -DRB1 disease loci. Hum Mol Genet 10:881-889

6. Bell GI, Horita S, Karam JH (1984) A polymorphic locus near the human insulin gene is associated with insulin-dependent diabetes mellitus. Diabetes 33:176-183

7. Barratt BJ, Payne F, Lowe CE et al (2004) Remapping the insulin gene/IDDM2 locus in type 1 diabetes. Diabetes 53: $1884-1889$ 
8. Nistico L, Buzzetti R, Pritchard LE et al (1996) The CTLA-4 gene region of chromosome $2 \mathrm{q} 33$ is linked to, and associated with, type 1 diabetes. Hum Mol Genet 5:1075-1080

9. Ueda H, Howson JM, Esposito L et al (2003) Association of the T-cell regulatory gene CTLA4 with susceptibility to autoimmune disease. Nature 423:506-511

10. Bottini N, Musumeci L, Alonso A et al (2004) A functional variant of lymphoid tyrosine phosphatase is associated with type I diabetes. Nat Genet 36:337-338

11. Smyth D, Cooper JD, Collins JE et al (2004) Replication of an association between the lymphoid tyrosine phosphatase locus (LYP/PTPN22) with type 1 diabetes, and evidence for its role as a general autoimmunity locus. Diabetes 53:3020-3023

12. Qu H, Tessier MC, Hudson TJ, Polychronakos C (2005) Confirmation of the association of the R620W polymorphism in the protein tyrosine phosphatase PTPN22 with type 1 diabetes in a family based study. J Med Genet 42:266-270

13. Onengut-Gumuscu S, Ewens KG, Spielman RS, Concannon $P$ (2004) A functional polymorphism (1858C/T) in the PTPN22 gene is linked and associated with type I diabetes in multiplex families. Genes Immun 5:678-680

14. Criswell LA, Pfeiffer KA, Lum RF et al (2005) Analysis of families in the multiple autoimmune disease genetics consortium (MADGC) collection: the PTPN22 620W allele associates with multiple autoimmune phenotypes. Am J Hum Genet 76:561-571

15. Ladner MB, Bottini N, Valdes AM, Noble JA (2005) Association of the single nucleotide polymorphism C1858T of the PTPN22 gene with type 1 diabetes. Hum Immunol 66:60-64

16. Zheng W, She JX (2005) Genetic association between a lymphoid tyrosine phosphatase (PTPN22) and type 1 diabetes. Diabetes 54:906-908

17. Zhernakova A, Eerligh P, Wijmenga C, Barrera P, Roep BO, Koeleman BP (2005) Differential association of the PTPN22 coding variant with autoimmune diseases in a Dutch population. Genes Immun 6:459-461

18. Kyogoku C, Langefeld CD, Ortmann WA et al (2004) Genetic association of the R620W polymorphism of protein tyrosine phosphatase PTPN22 with human SLE. Am J Hum Genet 75: 504-507

19. Begovich AB, Carlton VE, Honigberg LA et al (2004) A missense single-nucleotide polymorphism in a gene encoding a protein tyrosine phosphatase (PTPN22) is associated with rheumatoid arthritis. Am J Hum Genet 75:330-337

20. Velaga MR, Wilson V, Jennings CE et al (2004) The codon 620 tryptophan allele of the lymphoid tyrosine phosphatase (LYP) gene is a major determinant of Graves' disease. J Clin Endocrinol Metab 89:5862-5865

21. Gjorloff-Wingren A, Saxena M, Williams S, Hammi D, Mustelin T (1999) Characterization of TCR-induced receptorproximal signaling events negatively regulated by the protein tyrosine phosphatase PEP. Eur J Immunol 29:3845-3854

22. Cloutier JF, Veillette A (1999) Cooperative inhibition of T-cell antigen receptor signaling by a complex between a kinase and a phosphatase. J Exp Med 189:111-121

23. Gepts W (1965) Pathologic anatomy of the pancreas in juvenile diabetes mellitus. Diabetes 14:619-633

24. Roep BO, Arden SD, de Vries RR, Hutton JC (1990) T-cell clones from a type-1 diabetes patient respond to insulin secretory granule proteins. Nature 345:632-634

25. Ziegler AG, Hummel M, Schenker M, Bonifacio E (1999) Autoantibody appearance and risk for development of childhood diabetes in offspring of parents with type 1 diabetes: the 2-year analysis of the German BABYDIAB Study. Diabetes 48:460-468

26. Kukko M, Kimpimaki T, Korhonen S et al (2005) Dynamics of diabetes-associated autoantibodies in young children with human leukocyte antigen-conferred risk of type 1 diabetes recruited from the general population. J Clin Endocrinol Metab 90:2712-2717
27. LaGasse JM, Brantley MS, Leech NJ et al (2002) Successful prospective prediction of type 1 diabetes in schoolchildren through multiple defined autoantibodies: an 8-year follow-up of the Washington State Diabetes Prediction Study. Diabetes Care 25:505-511

28. Vandewalle CL, Falorni A, Lernmark A et al (1997) Associations of GAD65- and IA-2- autoantibodies with genetic risk markers in new-onset IDDM patients and their siblings. The Belgian Diabetes Registry. Diabetes Care 20:1547-1552

29. Sabbah E, Savola K, Kulmala P et al (1999) Disease-associated autoantibodies and HLA-DQB1 genotypes in children with newly diagnosed insulin-dependent diabetes mellitus (IDDM). The Childhood Diabetes in Finland Study Group. Clin Exp Immunol 116:78-83

30. Graham J, Hagopian WA, Kockum I et al (2002) Genetic effects on age-dependent onset and islet cell autoantibody markers in type 1 diabetes. Diabetes 51:1346-1355

31. Genovese S, Bonfanti R, Bazzigaluppi E et al (1996) Association of IA-2 autoantibodies with HLA DR4 phenotypes in IDDM. Diabetologia 39:1223-1226

32. Hermann R, Laine AP, Veijola R et al (2005) The effect of HLA class II, insulin and CTLA4 gene regions on the development of humoral beta cell autoimmunity. Diabetologia 48:1766-1775

33. Kupila A, Muona P, Simell T et al (2001) Feasibility of genetic and immunological prediction of type I diabetes in a population-based birth cohort. Diabetologia 44:290-297

34. Alberti KG, Zimmet PZ (1998) Definition, diagnosis and classification of diabetes mellitus and its complications. Part 1: diagnosis and classification of diabetes mellitus provisional report of a WHO consultation. Diabet Med 15:539-553

35. Kiviniemi M, Nurmi J, Turpeinen $H$, Lovgren $T$, Ilonen J (2003) A homogeneous high-throughput genotyping method based on competitive hybridization. Clin Biochem 36:633-640

36. Sjoroos M, Iitia A, Ilonen J, Reijonen H, Lovgren T (1995) Triple-label hybridization assay for type-1 diabetes-related HLA alleles. Biotechniques 18:870-877

37. Nejentsev S, Sjoroos M, Soukka T et al (1999) Populationbased genetic screening for the estimation of type 1 diabetes mellitus risk in Finland: selective genotyping of markers in the HLA-DQB1, HLA-DQA1 and HLA-DRB1 loci. Diabet Med 16:985-992

38. Laaksonen M, Pastinen T, Sjoroos M et al (2002) HLA class II associated risk and protection against multiple sclerosis-a Finnish family study. J Neuroimmunol 122:140-145

39. Haller K, Kisand K, Nemvalts V, Laine AP, Ilonen J, Uibo R (2004) Type 1 diabetes is insulin $-2221 \mathrm{MspI}$ and CTLA-4 +49 A/G polymorphism dependent. Eur J Clin Invest 34: $543-548$

40. Kimpimaki T, Kulmala P, Savola K et al (2002) Natural history of beta-cell autoimmunity in young children with increased genetic susceptibility to type 1 diabetes recruited from the general population. J Clin Endocrinol Metab 87:4572-4579

41. Ronkainen MS, Hamalainen AM, Koskela P, Akerblom HK, Knip M (2001) Pregnancy induces nonimmunoglobulin insulinbinding activity in both maternal and cord blood serum. Clin Exp Immunol 124:190-196

42. Williams AJ, Bingley PJ, Bonifacio E, Palmer JP, Gale EA (1997) A novel micro-assay for insulin autoantibodies. J Autoimmun 10:473-478

43. Savola K, Sabbah E, Kulmala P, Vahasalo P, Ilonen J, Knip M (1998) Autoantibodies associated with type I diabetes mellitus persist after diagnosis in children. Diabetologia 41:1293-1297

44. Savola K, Bonifacio E, Sabbah E et al (1998) IA-2 antibodiesa sensitive marker of IDDM with clinical onset in childhood and adolescence. Childhood Diabetes in Finland Study Group. Diabetologia 41:424-429

45. Dudbridge F (2003) Pedigree disequilibrium tests for multilocus haplotypes. Genet Epidemiol 25:115-121

46. Undlien DE, Friede T, Rammensee HG et al (1997) HLAencoded genetic predisposition in IDDM: DR4 subtypes may be associated with different degrees of protection. Diabetes 46:143-149 
47. Walter M, Albert E, Conrad M et al (2003) IDDM2/insulin VNTR modifies risk conferred by IDDM1/HLA for development of type 1 diabetes and associated autoimmunity. Diabetologia 46:712-720

48. Van der Auwera BJ, Vandewalle CL, Schuit FC et al (1997) CTLA-4 gene polymorphism confers susceptibility to insulindependent diabetes mellitus (IDDM) independently from age and from other genetic or immune disease markers. The Belgian Diabetes Registry. Clin Exp Immunol 110:98-103
49. Hermann R, Bartsocas CS, Soltesz G et al (2004) Genetic screening for individuals at high risk for type 1 diabetes in the general population using HLA Class II alleles as disease markers. A comparison between three European populations with variable rates of disease incidence. Diabetes Metab Res Rev 20:322-329

50. Cucca F, Goy JV, Kawaguchi Y et al (1998) A male-female bias in type 1 diabetes and linkage to chromosome $\mathrm{Xp}$ in $\mathrm{MHC}$ HLA-DR3-positive patients. Nat Genet 19:301-302 